\title{
Push-Pull FEL, a new ERL concept th
}

\author{
Andrew Hutton* \\ Jefferson Lab, 12000 Jefferson Avenue, Newport News, VA 23606, USA
}

Available online 16 November 2005

\begin{abstract}
This proposal uses two sets of superconducting cavities with two identical electron beams going in opposite directions. Each set of superconducting cavities accelerates one electron beam and decelerates the other beam. This arrangement allows the energy used to accelerate one beam to be recovered and used again for the other beam. The difference between this proposal and other energy-recovery proposals is that each electron beam is decelerated by a different structure than that which accelerated it so this is energy exchange rather than energy recovery.

If the superconducting cavities produce sufficient energy, a further simplification can occur. In this arrangement, the superconducting cavities are contained within the optical resonator with the light pulses traversing them. This arrangement leads to an extremely compact layout suitable for a university laboratory.
\end{abstract}

(C) 2005 Elsevier B.V. All rights reserved.

PACS: 80

Keywords: Energy recovery; Recirculating linacs; Free electron lasers; Radio frequency; Superconductivity

\section{Introduction}

This paper describes an ultra-compact high-power free electron laser that could make available tunable coherent light to a wide range of universities and small research institutes.

The principle is based on the energy-recovered Free Electron Laser (FEL) at Jefferson Lab [1] in which an electron beam is accelerated in superconducting cavities, traverses a wiggler to produce light pulses, and is brought back to the beginning of the superconducting cavities at a phase that decelerates the electrons returning their energy to the cavities. The light pulses from the wiggler are contained in an optical resonator (a pair of mirrors spaced by a precise multiple of the bunch separation) that reflects the light back to the wiggler in synchronism with a later electron bunch to provide stimulated, amplified light emission.

\footnotetext{
DOE contract number: DE-AC05-84ER 40150

*Tel.: + 17572697396 ; fax: + 17572695519.

E-mail address: andrew@jlab.org.
}

The main driver behind the new concept was the decision by the International Technology Recommendation Panel in August 2004 to select superconducting RF technology for the ILC. The technology selected is similar (but not identical) to that developed for the X-FEL at DESY [2], and many components are similar. This has initiated a process that will lead, rather quickly, to industrialization of most components required for a superconducting accelerator; in particular the cryomodules, the RF power sources, and the injector.

It therefore seems reasonable to re-examine the cost optimization of an FEL based on "cheap" industrial ILC components. This modifies the design of the electron optics in favor of more cryomodules, more injectors, and less beam transport.

This proposal uses two sets of superconducting cavities with two identical electron beams going in opposite directions. Each set of superconducting cavities accelerates one electron beam and decelerates the other beam. This layout allows the energy used to accelerate one beam to be recovered and used again for the other beam. The difference between this proposal and other energy-recovering 
proposals is that each electron beam is decelerated by a different structure than that which accelerated it, so this is energy exchange rather than energy recovery. This is similar to the original energy recovery proposal by Tigner [3] for electron colliders.

If the superconducting cavities produce sufficient energy, a further simplification can occur. In this layout, the superconducting cavities are contained within the optical resonator with the light pulses traversing them. This arrangement leads to an extremely compact layout suitable for a university laboratory.

The layout is shown in Fig. 1. The two cryomodules containing the superconducting cavities flank a wiggler that is used to produce coherent light. The addition of a pair of mirrors outboard of the cryomodules completes the FEL optical cavity. On either end, there is a $\sim 10 \mathrm{MeV}$ injector, which can either be a copy of that used at the Jefferson Lab FEL, an SRF gun, or whatever gun design is industrialized for the ILC. The electron beams are brought onto the acceleration axis by a bend magnet, which also serves to bend the spent beam to a dump. This bend magnet needs to be carefully designed to transport electrons with a large $(\sim 50 \%)$ energy spread to the dump with extremely small losses.

\section{Concept of operation}

The facility operates as follows. An electron bunch leaves the left gun, is accelerated by a superconducting cavity (these two elements and some pre-bunching elements constitute the left injector) and is brought onto the axis of the cryomodule by the left separator magnet. The bunch is then accelerated by the left cryomodule into the wiggler where it emits light; decelerated in the right cryomodule; and deflected into the dump by the right separator magnet (Fig. 2). The light pulse travels with the electron bunch but is not deflected by the right separator magnet so it continues on to the mirror. Most of the light is reflected back along the cryomodule axis, while a small fraction exits the optical resonator as a coherent laser pulse.

A second electron bunch leaves the right gun, is accelerated by the superconducting cavity and is brought onto the axis of the cryomodule by the right separator magnet, in synchronism with the reflected light pulse. The second bunch is accelerated by the right cryomodule, arriving at the wiggler at the same time as the light pulse so that light amplification can occur. The electron bunch is then decelerated in the left cryomodule and is deflected into the dump by the left separator magnet (Fig. 3). The light pulse is not deflected by the separator magnet and continues on to the mirror where it is reflected back along the cryomodule axis ready to meet the next bunch from the left gun. Looking at the energy balance, the energy in the cryomodules is recovered completely, while the energy given to the beam by the injector is partially converted to FEL light and partially dissipated in the dump. So the maximum FEL power that can be extracted is some fraction (up to about $50 \%$ ) of the power in the injector.

\section{Parameter example}

An example of a parameter set has been calculated based on the design parameters of the Jefferson Lab $10 \mathrm{~kW}$ FEL. Since the FEL has now achieved its design power output, the parameters are within the state of the art.

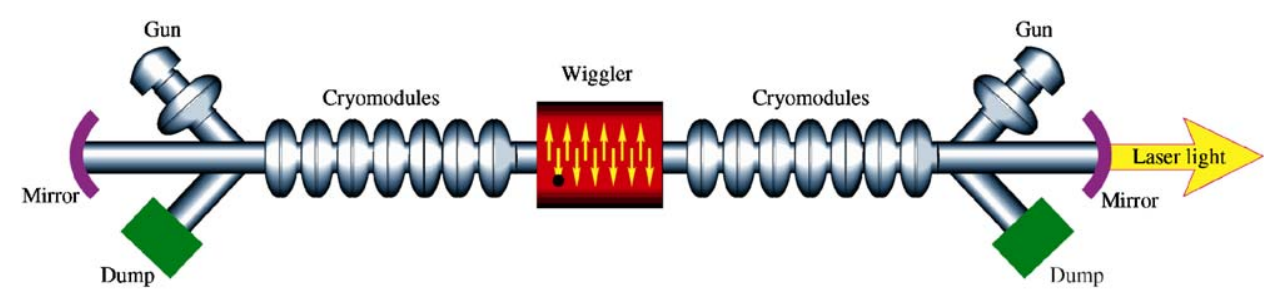

Fig. 1. Conceptual layout of the Push-Pull FEL.

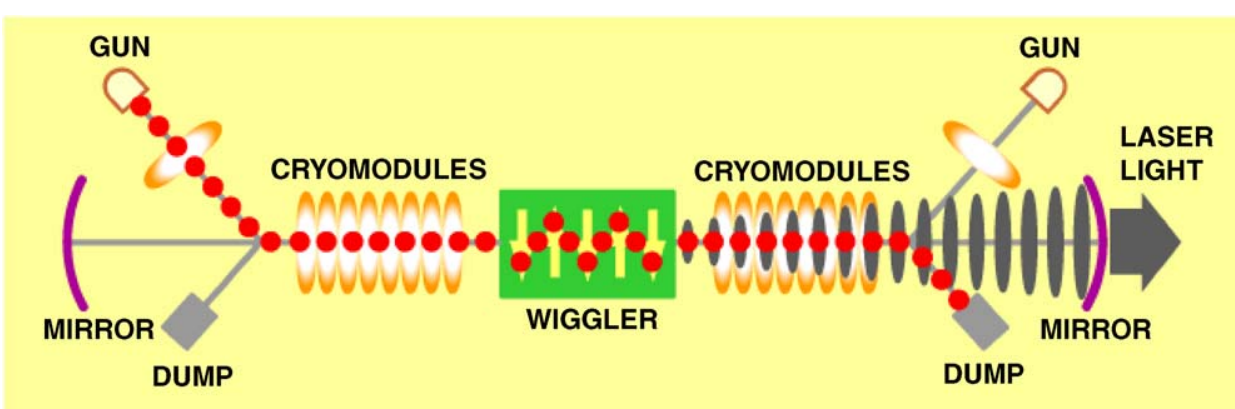

Fig. 2. Electron and light paths left-to-right. 


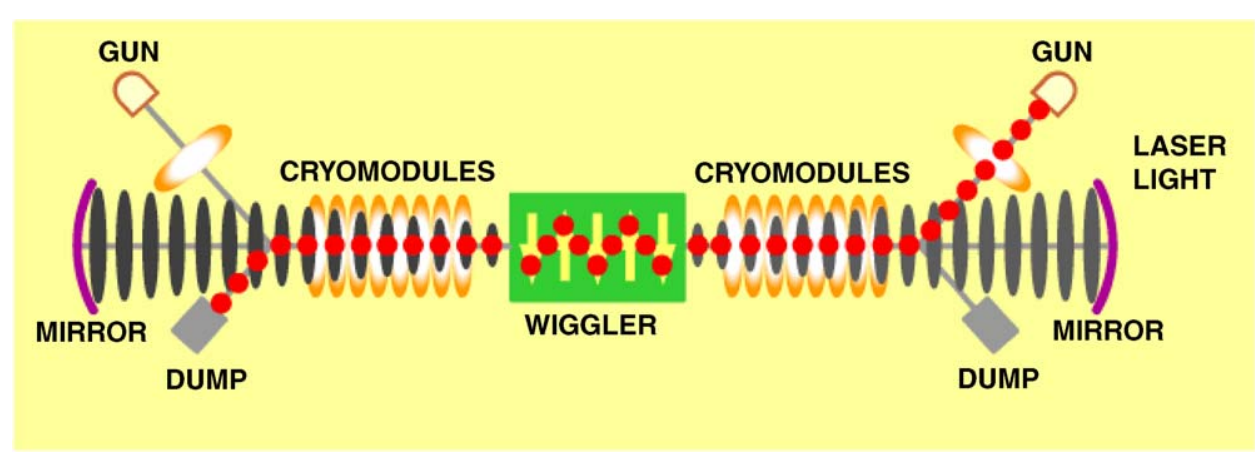

Fig. 3. Electron and light paths right-to-left.

Table 1

Example parameter set

\begin{tabular}{lll}
\hline Parameter & $10 \mathrm{~kW}$ JLab FEL design & Push-Pull FEL design \\
\hline Maximum beam energy & $80-210 \mathrm{MeV}$ & $200 \mathrm{MeV}$ \\
Injector beam energy & $10 \mathrm{MeV}$ & $10 \mathrm{MeV}$ \\
Beam current & $10 \mathrm{~mA}$ & $2 \times 0.5 \mathrm{~mA}$ \\
Beam power & $800-2100 \mathrm{~kW}$ & $2 \times 100 \mathrm{~kW}$ \\
Non-recovered beam power & $100 \mathrm{~kW}$ & $2 \times 5 \mathrm{~kW}$ \\
RF frequency & $1500 \mathrm{MHz}$ & $1300 \mathrm{MHz}$ \\
FEL repetition rate & $3.9-125 \mathrm{MHz}$ & $5.078 \mathrm{MHz}$ \\
& $\mathrm{RF}$ frequency $/(4-384)$ & $\mathrm{RF}$ frequency $/ 256$ \\
Optical cavity length & $32 \mathrm{~m}$ & $29.539 \mathrm{~m}$ \\
Bunch charge & $135 \mathrm{pC}$ at $75 \mathrm{MHz}$ & $100 \mathrm{pC}$ \\
Energy spread after wiggler & $10 \%$ of $210 \mathrm{MeV}$ & $2.5 \%$ of $200 \mathrm{MeV}$ \\
Energy spread at dump & $\sim 2 \%$ of $10 \mathrm{MeV}$ & $50 \%$ of $10 \mathrm{MeV}$ \\
FEL output power & $10 \mathrm{~kW}$ in the Infrared $1 \mathrm{~kW}$ in the UV & $>1 \mathrm{~kW}$ in the UV with bunch compression \\
\hline
\end{tabular}

The superconducting accelerating system is based on DESY X-FEL prototypes. The cryomodule contains eight 9-cell superconducting cavities operating at $23 \mathrm{MV} / \mathrm{m}$ for a total of $190 \mathrm{MV}$. The superconducting cavity in the injector is one of the same cavities, which is operated at less than $10 \mathrm{MV} / \mathrm{m}$ because the beam current will be higher. In any case, the injection energy should be less than $\sim 10 \mathrm{MV}$ to avoid neutron activation of the dump (the energy recovery process in the Linacs requires that the maximum energy at the dump be equal to the energy of the Injector). The bunch current is assumed to be equal to that used in the Jefferson Lab $10 \mathrm{~kW}$ FEL so no parameter extrapolation is required. The bunch repetition rate $(2 \times 5 \mathrm{MHz})$ is determined by the distance between the mirrors, which is assumed to be around $30 \mathrm{~m}$, and the requirement to be a sub-harmonic of the RF frequency $(1300 \mathrm{MHz})$. Note that in this scenario there are two bunches, one from each gun, in every round trip of the light. So the FEL light output is at $5 \mathrm{MHz}$. The other parameters are taken almost exclusively from the Jefferson Lab $10 \mathrm{~kW}$ FEL so they are all achievable in routine operation (Table 1 ).

\section{Light output}

The light output has been estimated by Benson [4] using his code [5] for two cases. The first assumes that the electron bunches are not compressed, which gives the most compact system. Since the energy is $200 \mathrm{MeV}$ and the charge is $100 \mathrm{pC}$ the obvious application for the driver is for a UV laser. A wiggler design that worked well for the UV and for a $200 \mathrm{MeV}$ beam was used, which is an Undulator A from ANL. It is already designed so it fits in well with the design philosophy. With this wiggler and a 2 ps FWHM bunch length there is a gain of about $60 \%$ in the UV and the power estimated by the spreadsheet is a few hundred watts (see Fig. 4). It should be noted that since the bunch is so long, the spreadsheet assumption that there is a single super-mode breaks down so the power may be much higher.

The second scheme assumes a buncher/debuncher system which can bunch down to $\frac{1}{3}$ of a ps FWHM. This gives very high gain of several hundred percent. The power is also estimated to be more than $1 \mathrm{~kW}$ (see Fig. 5). This is high enough that the power limit will be the optics and not the electron beam.

\section{Other hardware}

Much of the other hardware can be taken from the ILC or the DESY X-FEL to avoid costly development work. The $1300 \mathrm{MHz} 150 \mathrm{~kW}$ CW klystron which is being developed at Cornell for the ERL Light Source would be perfect for the RF Power source. It would have enough power for one injector and one cryomodule, so two will be 

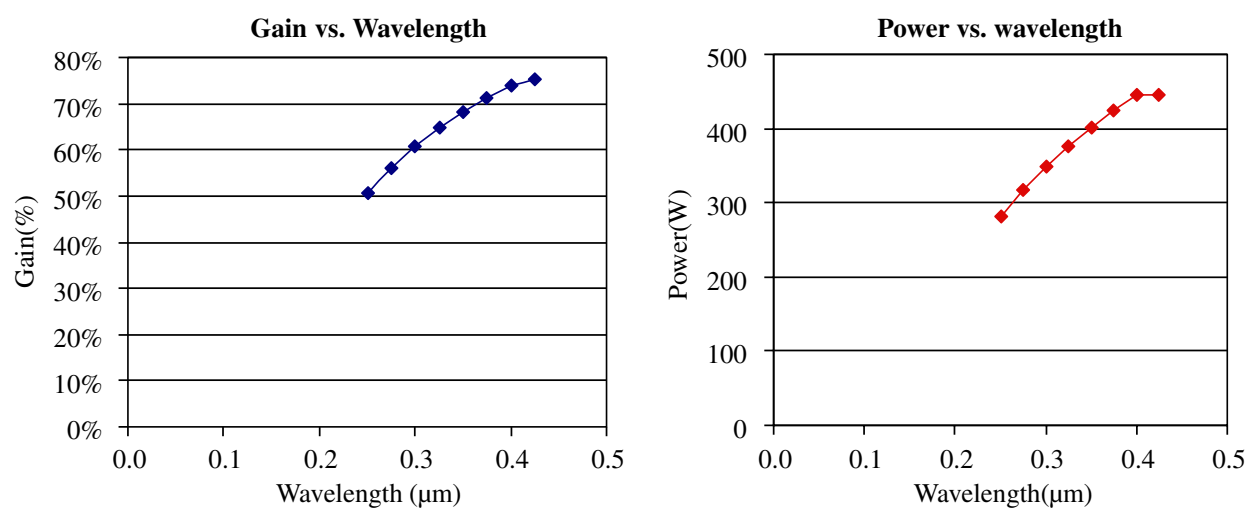

Fig. 4. FEL gain and output power as a function of wavelength with nominal bunch length.
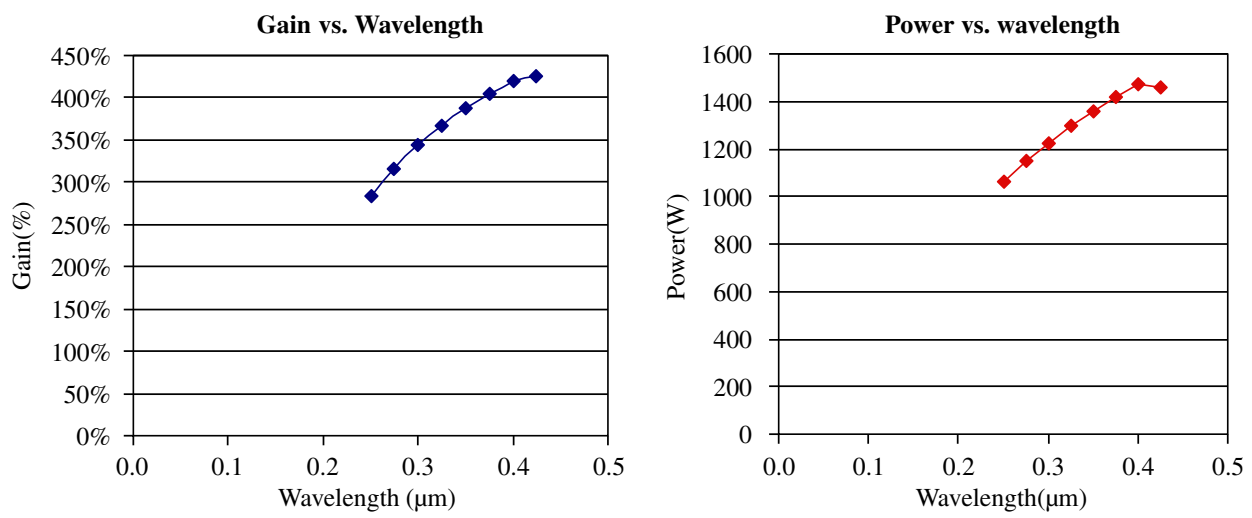

Fig. 5. FEL gain and output power as a function of wavelength with bunch compression.

required. Similarly, the RF and LLRF controls being developed for the Cornell ERL Light Source would also be well matched to this application; they are at the correct frequency and are designed for $\mathrm{CW}$ operation (the RF is pulsed in the ILC and the DESY X-FEL). However, the RF power distribution system using power splitters and electronic matching to the cavities, which has been adopted for ILC can be used for CW operation without modification.

\subsection{Focusing}

The optics for the Push-Pull FEL will be based on a quadrupole doublet at each Injector and two quadrupole doublets each side of the wiggler. The optics is designed to focus a round beam at the cathode to a round beam at the center of the wiggler. Since a quadrupole that is vertically focusing for the accelerating beam will be vertically defocusing for the decelerating beam and vice versa, the vertical beta functions for the accelerating beam will be identical to horizontal beta functions of the decelerating beam and vice versa. So this optical design (evaluated in detail by Douglas [6]) automatically provides well behaved optics for both beams. It is well suited to the case of the beam with the nominal bunch length discussed above.

However, it was shown above that the output power can be considerably enhanced by using a chicane to bunch the beams more tightly. In this case, the edge focusing of the chicane bends is independent of the direction of the particle. This destroys the anti-symmetry of the simple focusing scheme and requires the use of chicane magnets with carefully shaped poles. This will be the subject of a later paper.

\section{Acknowledgments}

I have received innumerable suggestions for improvements and active assistance in developing this idea. In particular, Steve Benson and Dave Douglas offered their expert assistance to evaluate the light output and the electron optics, respectively. I would also like to thank Fred Dylla and George Neil for their support, and Gwyn Williams for discussions that were always helpful, and Tom Oren for his inventive illustrations.

\section{References}

[1] G.R. Neil, et al., Phys. Rev. Lett. 84 (4) (2000).

[2] R. Brinkmann, et al. (Eds.), Supplement to the TESLA XFEL TDR., DESY 2002-167, October 2002, http://xfel.desy.de

[3] M. Tigner, Nuovo Cimento 37 (1965) 1228.

[4] S. Benson, Private Communication.

[5] S. Benson, et al., Nucl. Instr. and Meth. A 429 (1999) 27.

[6] D. Douglas, Private Communication. 